\title{
Die doppelte Einbettung der Logistikarbeit und die Grenzen prekärer Beschäftigung
}

Die „logistische Revolution“ der 1990er und 20ooer Jahre fiel zeitlich mit einer Phase der Deregulierung und Prekarisierung der Arbeit zusammen. In dieser Zeit etablierte sich die Handelslogistik als beschäftigungsintensives Segment des tertiären Sektors, das besonders stark von Ungleichheiten geprägt ist. Doch das in dieser Branche dominierende Produktionsmodell wird mehr und mehr in Frage gestellt, weil sich Arbeitskräfteknappheit auch hier bemerkbar macht. Die Unternehmen reagieren mit neuen Rekrutierungsstrategien und Rationalisierungsanstrengungen - mit begrenztem Erfolg. Dadurch öffnet sich ein arbeitspolitisches Gelegenheitsfenster für die Verbesserung der Beschäftigungsbedingungen.

FLORIAN BUTOLLO, ROBERT KOEPP

\section{Dimensionen der Einbettung des Logistik-Sektors}

Allgemein ist die Ausweitung geringqualifizierter und schlecht entlohnter Tätigkeiten im Dienstleistungssektor ein wesentliches Merkmal der Arbeitsmarktentwicklung der letzten Jahre (Moody 2017, S. 23ff.; Staab 2014). Das „Produktionsmodell“ (vgl. Boyer/Freyssenet 2003) ${ }^{1}$ der Handelslogistik schöpfte aus einem reichlichen Angebot ungelernter Arbeit im sekundären Arbeitsmarktsegment. Wie in anderen Feldern einfacher Dienstleistungen auch hat sich die sozialstrukturelle Einbettung dieses Modells jedoch deutlich verändert. Mittlerweile haben die Unternehmen der Branche auch im Bereich der ungelernten Arbeit Probleme damit, Positionen zu besetzen, wofür sowohl demografische Ursachen als auch höhere subjektive Ansprüche der Beschäftigten verantwortlich sind. Die typische soziale Grundlage des Produktionsmodells - ein großes regionales Arbeitskraftreservoir - löst sich auf. Unser Beitrag beleuchtet basierend auf Fallstudien in der Handelslogistik, mit welchen Gegenmaßnahmen („Fixes“) die Unternehmen der Branche versuchen, ihr Produktionsmodell zu stabilisieren. Die Strategien der geografischen Ausweitung der Rekrutierung („sozialräumlicher Fix“) und der Rationalisierungsinvestitionen („technologischer Fix“) setzen auf eine Fortsetzung des etablierten prekären Beschäftigungssystems unter neuen Vorzeichen. Auf der anderen Seite entsteht aufgrund der steigenden Marktmacht der Beschäftigten ein arbeitspolitisches Gelegenheitsfenster für einen „arbeitspolitischen Fix“: die stabilere Bindung der Belegschaften zu besseren Konditionen. $^{2}$
1 Im Anschluss an Robert Boyer und Michel Freyssenet (2003, S. 43f.) bezeichnen wir damit die sozial eingespielte Konstellation in einem Unternehmen, seine Profitstrategie mittels Produktpolitik, Arbeitsorganisation und Arbeitsbeziehungen - zu verfolgen. Diese produktive Konstellation ist jeweils zumindest implizit zwischen Kapital, Arbeiter*innen und weiteren Gruppen zu einem sozialen Kompromiss ausgehandelt.

2 Unsere Argumentation beruht auf einer laufenden Datenerhebung im Rahmen eines Forschungsprojekts am
Weizenbaum-Institut für die vernetze Gesellschaft in Berlin. Wir streben dabei an, einen systematischen Überblick über die Zweige der deutschen Handelslogistik zu gewinnen. Wir greifen dabei zurück auf (1) eine Fallstudie I in einem ostdeutschen Logistiklager, die in Zusammenarbeit mit Kolleg*innen des Instituts für Soziologie der Universität Jena abgeschlossen wurde (zwölf leitfadengestützte Interviews mit Shopfloor-Beschäftigten und Softwareentwickler*innen, Werksbesichtigung), (2) eine laufende Fallstudie II zu einem ostdeutschen Verteilzentrum (bisher zwei leitfadengestützte Management-Interviews, Werksbesichtigung), (3) sechs 


\section{1 Ökonomische Einbettung: strategische Rolle und Merkmale des Sektors}

Logistik steht seit jeher in enger Verbindung mit der verarbeitenden Industrie. Die Entwicklung der Branche steht somit in enger Wechselwirkung zu deren Veränderungen, und obwohl sie statistisch mehrheitlich dem tertiären Sektor zugeordnet wird, ist ihre Verschränkung mit der industriellen Produktion derart eng, dass sie eine eigentümliche Zwischenstellung zwischen Industrie und Dienstleistung einnimmt. ${ }^{3}$

Wenn man sich diese Kopplung vor Augen hält, wird die „logistische Revolution“ (Vahrenkamp 2011) der 1990er Jahre fassbar, denn sie steht in engem Zusammenhang mit der zunehmenden Globalisierung und Flexibilisierung der Produktionsmodelle in der verarbeitenden Industrie hin zur Lean Production und einer Fertigung, die auf Nachfrageentwicklung reagiert (vgl. Coe 2014, S. 229 f.). Prägend waren hier die Fragmentierung vormals vertikal integrierter Fertigung und deren globale bzw. regionale Streuung, in deren Folge es zu einem starken Wachstum des Handels mit Zwischenprodukten kam. Marktseitig wurde die Logistik Voraussetzung für die schnelle Bedienung einer ausdifferenzierten Kundennachfrage, wobei das „Matching“ zwischen Anbietern und Kunden wie auch die Geschwindigkeit der Lieferung mehr und mehr zu entscheidenden Kriterien im Konkurrenzkampf wurden.

Logistische Kapazitäten sind die Voraussetzung für eine solche Beschleunigung und Flexibilisierung wirtschaftlicher Transaktionen, und somit nimmt der Sektor eine zunehmend strategische Rolle in der globalen Ökonomie ein (ebd., S. 225). Pfeiffer bezeichnet ihn als „Bindeglied globaler Wertschöpfungsketten“ (2016, S.198), und es ist anzunehmen, dass sich seine strategische Bedeutung als „bewegende Instanz der vierten industriellen Revolution“ (Ittermann/Eisenmann 2018, S. 62f.) in Zukunft noch steigern wird (vgl. BVL 2017, S. 5). Während einerseits die Bedeutung von Daten zur Koordinierung und Rationalisierung logistischer Operationen zunimmt (vgl. Coe 2014, S. 23of.), kann der Sektor andererseits auch als deren materielles Pendant betrachtet werden. Die strategische Herausforderung der Logistik ist mithin, Informations- und Stoffströme in Übereinstimmung zu bringen. Die wachsenden Volumina und die Beschleunigung der Transaktionen materialisieren sich in einer räumlichen Konzentration von Umschlagpunkten der logistischen Dienstleister an Verkehrsknotenpunkten und in der Nähe großer urbaner Ballungsräume, nicht selten mit zehntausenden Beschäftigten (vgl. Moody 2017, S. 59ff.).

Neben dem Transport gehören Lagerkapazitäten zum Rückgrat der logistischen Revolution. Diese Orte, an denen das Umsortieren, Kommissionieren und Lagern von Gütern stattfindet, sind häufig Betriebe innerhalb einer Unternehmensfamilie oder werden von externen Dienstleistern betrieben. Seit den 199oer Jahren zeichnet sich ein Trend zur Vermarktlichung in der Beziehung zwischen
Handelsunternehmen und Betrieben der Lagerlogistik ab (vgl. Fernie/Sparks 2014, S. 6f.; Jaehrling 2019). Über vertragliche Beziehungen werden Leistungen und Preise zwischen den Händlern und den Betrieben festgelegt, und dadurch wird eine Fragmentierung der Lieferkette geschaffen, in deren Folge Logistikbetriebe in eine untergeordnete Rolle geraten. Somit spitzt sich die Auseinandersetzung zwischen Handelsunternehmen, Produzenten und den Logistikbetrieben um die Distribution des Gesamtprofits zu (vgl. Newsome et al. 2013) und wird maßgeblich von den Unternehmen mit hoher Marktmacht geformt.

In der Handelslogistik, auf die wir uns im Folgenden konzentrieren, dominieren drei charakteristische Profitstrategien (vgl. Boyer/Freyssenet 2003, S.36ff.). Erstens versuchen die Unternehmen der Branche, den Umschlag, also die Volumina, zu erhöhen, während sie zweitens fortwährend Prozessinnovationen vorantreiben, um Kosten einzusparen. Drittens ist die Wettbewerbsposition von Unternehmen zunehmend davon abhängig, wie flexibel sie in der Distribution auf schwankende Kundensignale reagieren können. Entsprechend haben Händler und Logistiker ihre Rationalisierungsstrategien an Lean-Prinzipien bzw. Prinzipien des agilen Managements ausgerichtet, um Effizienz, Umschlagerweiterung und Flexibilität zu erhöhen (Fernie/Sparks 2014, S. 8ff.). Das betriebliche Problem, flexibel auf Marktschwankungen reagieren zu können, wird in der Handelslogistik nicht zuletzt über die Gestaltung der Beschäftigungsverhältnisse bearbeitet. Der nächste Abschnitt wird vor allem diese Säule des Produktionsmodells der Handelslogistik diskutieren.

\subsection{Sozialstrukturelle Einbettung: \\ Das Beschäftigungssystem der Handelslogistik}

Die Handelslogistik ist nach Beschäftigung und Umsatz ein gewichtiger Teil des Logistiksektors. In einer großzügigen Schätzung gehen Ittermann und Eisenmann (2018, S.6o) davon aus, dass ca. 2,95 Mio. Beschäftigte direkt oder indirekt der Logistikbranche zuzuordnen sind. Darunter fallen Beschäftigte mit Aufgaben in Lagerei, Umschlag, Transport und Administration. Der Bundesvereinigung Logistik zufolge sind $2011 \mathrm{im}$ Handel bundesweit 64 Mrd. $€$ an Logistikkosten angefallen. Diese Summe entspricht einem Anteil von ca. 30\% der gesamtwirtschaft-

Expertengespräche (Ministeriumsmitarbeiter*innen eines ostdeutschen Bundeslandes, hauptamtliche Gewerkschafter*innen, Branchenexperten), die einen Branchenüberblick gegeben haben, sowie umfangreiches Sekundärmaterial.

3 In sozialtheoretischer Perspektive ordnet beispielsweise Moody (2017, S. 62) den Sektor schlicht der (wertschöpfenden) Produktion zu, da diese - angelehnt an Marx' Formulierungen in seinem Werk "Grundrisse der Kritik der politischen Ökonomie" - alle Prozesse der Stoffumwandlung und des Transports zum Ort der Konsumtion beinhalte. 
lichen Logistikkosten (vgl. Seeck et al. 2014, S.16). Auch wenn Logistik als „Kernkompetenz des Handels“ (Jaehrling et al. 2018, S. 295; Zanker 2018, S. 21) bezeichnet wird, können die Logistikfunktionen nicht nur inhouse durch die Handelsunternehmen selbst, sondern auch extern durch spezialisierte Unternehmen z. B. der Lagerei ausgeführt werden. ${ }^{4}$

Logistikarbeit umfasst „vielfach ein Beschäftigungssegment, das durch niedrige Qualifikationen, kurzfristige Anlernzeiten sowie routinisierte und repetitive Tätigkeiten gekennzeichnet ist - verschiedentlich auch als Einfacharbeit charakterisiert" (Hirsch-Kreinsen et al. 2018). Dies spiegelt sich in der Beschäftigungsstatistik der Bundesagentur für Arbeit wider (BA 2019b). ${ }^{5}$ Die Berufsgattungen in der „Lagerwirtschaft, Post und Zustellung bzw. Güterumschlag" machen den größten Teil der Beschäftigung in der Handelslogistik aus. Im Dezember 2018 arbeiteten 2,375 Mio. Beschäftigte in dieser Berufsgattung, wovon $27,3 \%$ geringfügig und $72,7 \%$ sozialversicherungspflichtig beschäftigt waren (vgl. Abbildung 1). Obwohl unter den sozialversicherungspflichtig Beschäftigten gut die Hälfte einen Abschluss in der beruflichen Bildung besaß, arbeiteten $78 \%$ davon in Helfertätigkeiten. Unter den geringfügig Beschäftigten arbeiteten sogar neun von zehn in un- und angelernten Tätigkeitsbereichen. Dieses Missverhältnis zwischen vorhandenen Ausbildungen und faktischen Helferfunktionen legt nahe, dass viele der berufsfachlichen Qualifikationen der Logistikarbeiterinnen in der Branche keine Verwendung finden.

Entgeltdaten unterstreichen das unterdurchschnittliche Lohnniveau in der Logistikbranche, in der ein Viertel der Beschäftigten dem Niedriglohnsegment zuzuordnen ist (Zanker 2018, S. 155). Die Helfertätigkeit in der Lagerwirtschaft in Vollzeit erbrachte 2017 einen durchschnittlichen Monatsbruttolohn von $2183 €$, eine Kauffrau bzw. ein Kaufmann im Einzelhandel verdiente im Durchschnitt $2390 €$ und eine Fachkraft im Großhandel $3067 €$ (vgl. BA 2019c). ${ }^{6}$ Generell ist bei diesen Zahlen zu berücksichtigen, dass sich dahinter ein deutliches West-Ost- sowie SüdNord-Gefälle der Löhne verbirgt.

Die großen Logistikwerke und Distributionszentren sind arbeitsintensive Einrichtungen mit hunderten bis

\section{ABBILDUNG 1}

\section{Entwicklung der Beschäftigung im stationären} Kernbereich der Handelslogistik', 2013-2018

Angaben in Millionen

Sozialversicherungspflichtig Beschäftigte

Geringfügig Beschäftigte

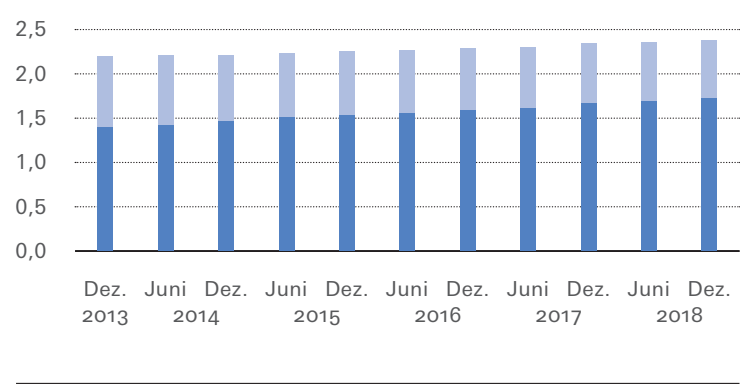

A Berufe in Lagerwirtschaft, Post und Zustellung bzw. Güterumschlag (Gruppe 513 der Klassifikation der Berufe)

Quelle: BA (2019b)

tausenden von Mitarbeiter*innen. Die Ausrichtung auf Effizienz und Flexibilität bei relativ geringen Gewinnmargen strukturiert die Beschäftigungsverhältnisse der Logistikbranche unter der Bedingung begrenzter institutioneller und geringer Organisationsmacht der Belegschaften. In Lagerei, Güterumschlag und Paketdistribution können sie überwiegend als offene Beschäftigungssysteme ${ }^{7}$ charakterisiert werden, da die Beschäftigung überwiegend auf kurze und mittlere Dauer ausgelegt ist und somit eine hohe Rotation zwischen betrieblichem Beschäftigungssystem und Arbeitsmarkt stattfindet. Im Segment der Handelslogistik erfüllen solche Beschäftigungssysteme den Zweck, flexibel auf die Auftragsschwankungen der Produktmärkte reagieren zu können, indem sie Personal je nach Auftragslage zeitweise ab- oder aufbauen (vgl. Bonacich/Wilson 2008, S. 15; Jaehrling et al. 2018, S. 308). Die Volatilität der Märkte wird in Form sozialer Unsicherheit auf die Beschäftigten abgewälzt, die als „Kontingenzarbeitskräfte“ (Staab/Nachtwey 2015) zum Flexibilitätspuffer der Branche werden.
4 Noch vor Berufskraftfahrern (30\%) stellen Arbeitskräfte mit Aufgaben in der Lagerwirtschaft, im Güterumschlag und der Zustellung die größte Beschäftigtengruppe (40\%) in einem breit definierten Verständnis von Logistik (Zanker 2018, S. 117).

5 Wenn nicht anders ausgewiesen, speisen sich die folgenden Aussagen aus eigenen Berechnungen auf Grundlage der Daten der Beschäftigungsstatistik der Bundesagentur für Arbeit (BA 2019b). Wir werden uns im Folgenden auf diese Berufsgattung (Gruppe 513 der Klassifikation der Berufe) beziehen, da sie am ehesten den stationären Kernbereich der Handelslogistik aus Distributions-, Logistik- und Verteilzentren, Lagerhäusern und der Zustellung repräsentiert.
6 Die Tariflandschaft in der Handelslogistik ist fragmentiert Die Tarifverträge aus dem Einzel- und Großhandel bieten vergleichsweise bessere Bedingungen als der Tarifvertrag "Logistik".

7 Beschäftigungssysteme sind „Strategien, mit denen Betriebe zwei grundlegende Bezugsprobleme der Personalpolitik bearbeiten: (a) auch bei hoher Diskontinuität im Arbeitsvolumen die Verfügbarkeit qualifizierten Personals sicher zu stellen und (b) dessen Leistungsbereitschaft zu gewährleisten [...]." (Krause 2012, S.122) 
Die Voraussetzung dafür ist das deutliche Übergewicht ungelernter Aufgabenprofile, für welche Arbeiter*innen aus dem sekundären Arbeitsmarkt rekrutiert werden und in relativ kurzer Zeit angelernt werden können. Aufgrund der körperlichen Beanspruchung, dem geringen Lohn und mangelnder Aufstiegschancen im internen Arbeitsmarkt ist die Branche zudem von einer sehr hohen Fluktuation der Belegschaften geprägt (vgl. Min 2007). ${ }^{8}$ Bei einem von uns untersuchten Versandhändler „öffnen“ die Personalabteilungen die Beschäftigungssysteme, indem sie z.B. prinzipiell nur befristet einstellen, befristete Verträge bis zur maximalen Länge von zwei Jahren stückeln oder Leiharbeit einsetzen. Die Fluktuation ist somit bereits in den Grundzügen der Personalpolitik angelegt. Diese Praktiken sind in der Branche weit verbreitet. ${ }^{9}$ Entsprechend sind Leiharbeitsfirmen im Logistiksektor ein wichtiger personalpolitischer Akteur. Laut Bundesagentur für Arbeit waren Ende 2016 mit rund 241 ooo Personen ein Viertel aller Leiharbeiter*innen in Deutschland in den Berufen der Lagerwirtschaft beschäftigt (vgl. Ittermann/Eisenmann 2018, S. 68).

Durch Dauerbefristungen und den Einsatz von Leiharbeit errichten die Unternehmen ein System von „Bewährungsproben“ für die prekär Beschäftigten (vgl. Dörre/Haubner 2012, S.84f.). Mithilfe von Feedback- bzw. Mitarbeitergesprächen (die teils auf einer digitalen Leistungsmessung beruhen), flexiblen Arbeitszeitanforderungen, Monitoring der Krankentage usw. entstehen wiederkehrende Prüfungssituationen für Befristete und Leiharbeiter*innen, die letztlich über Weiterbeschäftigung, Übernahme und Entfristung entscheiden (vgl. Barthel/ Rottenbach 2017; Jaehrling 2018; Staab/Nachtwey 2015). Angesichts knapper permanenter Stellen müssen die prekär Beschäftigten ständig ihre Leistung und ihr Wohlverhalten beweisen, wenn sie in die Stammbelegschaft aufrücken wollen. Die Befristung erweist sich damit als überlange Probezeit (vgl. Dörre/Haubner 2012, S. 84; Jaehrling et al. 2018, S.308).

Doch solche Produktionsmodelle werden erst unter spezifischen Bedingungen sozial stabil: Eine zentrale Voraussetzung für die Handelslogistik ist die Verfügbarkeit von Arbeitskräften im sekundären Arbeitsmarktsegment. Solche brachliegenden Arbeitskraftressourcen hat insbesondere die sukzessive oder disruptive Deindustrialisierung - unter anderem bedingt durch die Restrukturierung der ostdeutschen Wirtschaft ab 1990 - hinterlassen.

Die gezielte Ansiedlung von Logistikwerken in strukturschwachen Regionen ist Gegenstand zahlreicher Fallstudien. Briken/Taylor (2018) untersuchten systematisch den Arbeitsmarktkontext eines von Amazon betriebenen fulfilment centre in Süd-Wales, wo durch die Schließung der Kohleminen, die Verlagerung metallverarbeitender Betriebe und den Untergang der Textilindustrie ein Großteil der industriellen Arbeitsplätze verschwanden. Die Belegschaft des Amazon-Lagers rekrutiert sich nun aus dem überdurchschnittlich hohen Anteil von Transferleistungs- bezieher*innen und Arbeitslosen. Auch Mulholland/ Stewart (2014) analysierten einen großen Einzelhandelslogistiker in den britischen Midlands im Kontext einer sich wandelnden lokalen Wirtschaftsstruktur. Dort lässt der neue Dienstleistungsschwerpunkt frühere berufliche Qualifikationen verfallen und macht die Arbeit im Distributionszentrum $\mathrm{zu}$ einer der wenigen verbleibenden Optionen.

Auch in Deutschland sucht die Lagerlogistik neben einer guten infrastrukturellen Anbindung die soziale Einbettung eines „schwachen“ Arbeitsmarktkontexts. Besonders deutlich ist dies in den ostdeutschen Bundesländern, wo Standorte großer Distributionszentren der Einzelhändler und Logistikwerke des Online-Versandhandels sich an den Rändern von Ballungszentren wie Leipzig und Erfurt oder in der brandenburgischen Peripherie Berlins angesiedelt haben. Infolge der Deindustrialisierung dieser Regionen nach der Wiedervereinigung hatten Sachsen, Sachsen-Anhalt und Brandenburg noch 2015 Arbeitslosenquoten von 16 bis $18 \%$, während z.B. Bayern und Baden-Württemberg Quoten von 7 bis 7,4\% aufwiesen (BA 2019a; vgl. auch Dörre et al. 2013). Diese Umstände führten zu einem Überangebot von Arbeitskräften und einer geringen Anspruchshaltung unter den Beschäftigten, welche gute Voraussetzungen für die Etablierung offener Beschäftigungssysteme boten.

Die hohe Erwerbslosigkeit dort - wie auch in strukturschwachen Regionen Westdeutschlands - prägt zugleich den Charakter des innerbetrieblichen Klassenkompromisses. Aufgrund der geringen Marktmacht und des prekären Charakters der Beschäftigungssysteme ist das Kräfteverhältnis zwischen Kapital und Arbeit in der Handelslogistik oft von einer erheblichen Asymmetrie gekennzeichnet. Nur Beschäftigte in großen Betrieben können auf institutionelle Machtressourcen der Betriebsräte zurückgreifen, die bei kleineren Dienstleistern oftmals schlicht nicht existieren (vgl. Zanker 2018, 122f.). Prekäre Beschäftigungsverhältnisse und teils auch eine gewerkschaftsfeindliche Haltung des Managements machen die Organisierung der Belegschaften jedoch zu einer Herausforderung, wie z.B. die Kampagnen bei den Online-Versandhändlern Amazon und Zalando zeigen. Ein von uns interviewter Gewerkschaftssekretär klagte entsprechend, dass „Arbeiter- und Klassenbewusstsein" in seinem Fachbereich keine Selbstverständlichkeit sei. Eher herrschte die Dankbarkeit darüber vor, überhaupt einen Job zu haben, was sich auch in einer Unfähigkeit äußerte, in den Konflikt mit dem Ma-

8 Evidenzen für eine hohe Mitarbeiterfluktuation im Gewerbe fanden wir ebenso in der Betriebsfallstudie im Online-Versandhandel (Butollo et al. 2018), in einer Fallstudie in der Paketdistribution und in Expertengesprächen mit Vertreter*innen aus Gewerkschaften und Ministerien.

9 Fallstudie I und ähnliche Beschäftigungspolitik in Fallstudiell. 
nagement zu gehen. Die gewerkschaftliche Verankerung müsse erst durch lange Kampagnen gerade bei den neuen Online-Versandhändlern erarbeitet werden.

Dies verweist darauf, dass die Beschäftigungs- und Arbeitsverhältnisse in der Handelslogistik nicht ohne die „sekundäre Ausbeutung“ durch „staatlich legitimierte Disziplinierungsmechanismen" möglich wären (Dörre/Haubner 2012, S.71, 81f.). Erstens wurden durch das Arbeitsmarktregime nach den „Hartz-Gesetzen“ die Reproduktionskosten der Arbeitskraft erst auf das geringe Niveau gesenkt, das Beschäftigung in Logistikwerken überhaupt „attraktiv“ erscheinen lässt, und damit soziale Ungleichheit geschaffen und stabilisiert. Zweitens verringert der sanktionsbewährte Arbeitszwang die individuelle Marktmacht von Arbeitslosen genauso wie die von aktuell Beschäftigten (vgl. auch Dörre et al. 2013).

\section{Antworten auf das versiegende Arbeitskräftereservoir: Stabilisierung der Low Road oder Aufwertung der Arbeit?}

Das vorherrschende Produktionsmodell der Handelslogistik ist durch eine doppelte Einbettung in Wertschöpfungsnetzwerke einerseits und regionale Arbeitsmärkte andererseits gekennzeichnet. Damit ist es voraussetzungsvoll: Die dominierenden Formen der Beschäftigung können nur aufrechterhalten werden, wenn gering entlohnte „Kontingenzarbeitskräfte“ verfügbar sind.

Die Bedingungen dafür haben sich in jüngster Zeit deutlich verändert. Für die neuen Bundesländer haben drei Faktoren zu einer virulenten Arbeitskräfteknappheit beigetragen: Erstens schlägt sich dort der demografische Wandel in Verbindung mit Abwanderungstendenzen besonders stark nieder. Zweitens hat sich auch in den neuen Bundesländern seit 2005 die Entwicklung zur „prekären Vollerwerbsgesellschaft“ (Dörre et al. 2013, S.348ff.) durchgesetzt und die Beschäftigungsoptionen sind gewachsen (vgl. BA 2019a). Drittens haben Beschäftigte höhere subjektive Ansprüche an ihren Arbeitsplatz entwickelt (vgl. Goes et al. 2015; Schmalz et al. 2017). Dies äußerte sich in einer Zunahme von innerbetrieblichen Auseinandersetzungen, aber auch in einer abnehmenden Bereitschaft, schlecht entlohnte und prekäre Stellen anzunehmen. All das trägt zu einer Situation bei, in der Arbeitskraftressourcen - subjektive Bereitschaft und objektive Verfügbarkeit - knapper werden. Selbst die sogenannten sekundären Arbeitsmärkte trocknen zunehmend aus - jenes Reservoir, aus dem der Bedarf an Helfertätigkeiten in der Handelslogistik-Branche gedeckt wird. In einem terminologischen Spagat wird auch dieses Phänomen von Regionalregierungen und Unternehmen unter dem Schlagwort „Fachkräftemangel“ thematisiert. Eine Branchenstudie der Bundesvereinigung Logistik (BVL 2017) sowie zahlreiche Hinweise aus unserer laufenden Forschung über die Transformation der Beschäftigungssysteme in der Handelslogistik ${ }^{10}$ weisen darauf hin, dass der Arbeitskräftemangel von den Akteuren der Branche als gemeinsamer Problemhorizont geteilt wird.

Diese Situation droht das Produktionsmodell der Handelslogistik zu destabilisieren, worauf die Unternehmen mit einer Serie von Maßnahmen reagieren, die wir im Folgenden mit Bezugnahme auf die wissenschaftliche Literatur und Daten aus den erwähnten eigenen betrieblichen Studien und Expertenbefragungen zusammenfassen. Wir beobachten ein Dreieck aus sozialräumlichen, technologischen und arbeitspolitischen Lösungsversuchen („Fixes"). ${ }^{11}$ In der Praxis experimentieren Unternehmen oft mit Lösungsansätzen auf allen drei Gebieten. Dennoch steht der Pfad des sozialräumlichen und des technologischen Fixes in einer Spannung zum arbeitspolitischen Fix, da sie auch als Versuche gelesen werden können, die Arbeitskraftproblematik zu lösen, ohne dass den Beschäftigten qualitativ bessere Konditionen zugestanden werden.

\subsection{Sozialräumlicher Fix: Erweiterung des Einzugsgebiets}

Wegen der Engpässe auf den lokalen Arbeitsmärkten versuchen Unternehmen, zusätzliche Arbeitskräfte aus anderen Regionen anzuwerben (vgl. Wagner/Hassel 2017). Ein großer Online-Versandhändler (Logistikstandort B, Fallstudie I) macht aus der Not gar eine Tugend, indem sich das Unternehmen als „Multikulti-Company“ geriert. Die größten nicht-deutschen Beschäftigungsgruppen in diesem Unternehmen stellten Arbeitsmigrant*innen aus den grenznahen Regionen Polens, aus der Slowakei und zuletzt auch zunehmend aus Syrien, Afghanistan und Rumänien. Insgesamt waren es Mitarbeiter ${ }^{\star}$ innen aus 62 Nationen. Das Unternehmen ergriff gezielt Maßnahmen, um die Integration der Beschäftigten mit Migrationshintergrund in den Betrieb zu erleichtern. So wurden beispielsweise ein Gebetsraum für muslimische Beschäftigte eingerichtet und Sprachkurse mit Unterstützung des Bundesamts für Migration und Flüchtlinge angeboten. Der Arbeitsprozess selbst ist multilingual. Informationen werden standardmäßig sowohl in Deutsch als auch in Englisch und in

10 Die folgende Darstellung ist eine Synopse der inhaltsanalytischen Auswertung einer abgeschlossenen und einer laufenden Fallstudie sowie der sechs Experteninterviews, die in Fußnote 2 erwähnt wurden.

11 Wir entlehnen den Begriff „Fixes" bei David Harvey (2006) und Beverly Silver (2003). Sie bezeichnen damit Maßnahmen, mit denen Unternehmen versuchen, in einer krisenhaften Situation temporär die Kontrolle über die Arbeit wiederzuerlangen und Profitabilität wiederherzustellen. 
Polnisch kommuniziert. Flankierend zu solchen Integrationsmaßnahmen gibt es Bestrebungen, den Arbeitsprozess durch den Einsatz digitaler Assistenzsysteme, auch unter Rückgriff auf nonverbale Symboliken, weiter zu vereinfachen, um Sprachbarrieren zu überwinden. ${ }^{12}$ Der Zugriff vor allem auf die Arbeitskräfte Osteuropas ist in ostdeutschen Logistikstandorten übliche Praxis und wird über Leiharbeitsfirmen organisiert (vgl. Jaehrling et al. 2018, S.307f.). Der Standortleiter eines Brandenburger Verteilteilzentrums (Fallstudie II) mit mehr als 400 Beschäftigten antwortet auf die Frage, woher seine Leiharbeiterinnen kommen:

„Also wir fischen alle auf demselben Markt und der ist Polen. Und da fischt nicht nur [unser Standort], da fischt auch [Güterverkehrszentrum B]. Da sind sehr viele große Unternehmer, Konsolidierer [...], und wo holen die ihre Leiharbeiter her? Aus Polen."

Für den regulären Betrieb des Verteilzentrums ist Leiharbeit, und damit häufig die Beschäftigung polnischer Arbeiter*innen, essenziell geworden: Das eigene Personal reicht nicht aus, um die Schichten abzudecken, die standardmäßig mit Leiharbeit aufgestockt werden. In Extremfällen kann z.B. durch krankheitsbedingte Ausfälle und starke Auftragslage die Leiharbeitsquote auf $50 \%$ klettern. Der Personalleiter desselben Verteilzentrums stellt Polen als unerschöpfliches Arbeitskräftereservoir dar, gibt allerdings auch zu bedenken: „Irgendwann muss Polen doch auch leer sein".

\subsection{Technologischer Fix: Automatisierung in der Handelslogistik}

Als arbeitsintensives Beschäftigungsfeld mit hohen Anteilen an Routinetätigkeiten besitzt die Handelslogistik theoretisch ein hohes Automatisierungspotenzial. Tatsächlich präsentieren sich Leitunternehmen der Branche derzeit als Vorreiter rascher technischer Prozessinnovationen (vgl. Butollo et al. 2018), die sich nicht nur auf dem Feld der Automatisierungsanlagen, sondern auch im Bereich der algorithmischen Steuerung der Abläufe sowie der Anwendung von Künstlicher Intelligenz abspielen (Ittermann/Eisenmann 2018). ${ }^{13}$ Allerdings haben sich Automatisierungslösungen bislang nur in Teilbereichen, z. B. in der Paketsortierung oder der Lebensmitteldistribution, durchgesetzt. Es zeigen sich technische Schranken der Automatisierung, die ihre Ursache in der großen Unterschiedlichkeit der Güter und den damit verbundenen spezifischen Anforderungen an Transport, Lagerung und Kommissionierung haben (Wright/Lund 2006, S. 65). Da sich eine „Containerisierung“ der Güter in standardisierten Behältern oftmals aufgrund des Gebots verbietet, den Platz in den Lagern möglichst effizient zu nutzen, stoßen automatisierte Anlagen an Grenzen der Bearbeitung der eingelagerten Güter. ${ }^{14}$ Auch der Transport von Waren mittels selbstfahrender, von Künstlicher Intelligenz gesteuerter Transportfahrzeuge ist technologisch weniger trivial, als es futuristische Darstellungen suggerieren (Moody 2018, S. 13; Ortmann/Walker 2019).

Neben technischen Schranken stoßen Automatisierungsprojekte auch auf betriebliche Budgetrestriktionen. Aufgrund der strukturell niedrigen Profite in der Branche, der starken Konkurrenz und der Vermarktlichung der Wertschöpfungsnetzwerke stehen die Unternehmen unter hohem Druck zur unmittelbaren Refinanzierung der Investments (Jaehrling et al. 2018). Solange eine hohe Zahl von niedrig entlohnten Kontingenzarbeitskräften verfügbar war, blieb der Anreiz für Automatisierungsinvestitionen daher gering. Die Abhängigkeit der Automatisierungsinvestitionen vom Arbeitsmarktkontext wurde bei einem von uns untersuchten Handelslogistiker sichtbar. Standorte in westdeutschen Hochlohnregionen wiesen dort einen höheren Automatisierungsgrad auf als die ostdeutschen Standorte, da sich bestimmte Investitionen unter anderem erst bei einem höheren Lohnniveau auf dem regionalen Arbeitsmarkt lohnen.

Trotzdem - die zentralen Variablen der Automatisierungsinvestitionen unterliegen derzeit einem Wandel Neue technologische Möglichkeiten im Feld der flexiblen Automatisierung, des Internets der Dinge und der Künstlichen Intelligenz verschieben den Horizont dessen, was technologisch unmittelbar machbar ist. Zugleich sinken die Investitionskosten in Automatisierungslösungen aufgrund der zunehmenden Leistungsfähigkeit und des höheren Reifegrades digitaler Technologien (vgl. Brynjolfsson/McAfee 2014). Schließlich liefert der skizzierte Arbeitsmarktkontext auch Anreize zur (Teil-)Automatisierung, da die Lohnkosten im Verhältnis zu den Investitionskosten steigen. Aus diesen Gründen experimentieren insbesondere die technologieaffinen Unternehmen des Onlineversandhandels mit Automatisierungsansätzen, was mittelfristig deutliche Auswirkungen auf das Produktionsmodell in der Handelslogistik haben könnte. Viele Unternehmen befinden sich in einem Suchprozess, in dessen Rahmen Prozessinnovationen zur Substitution eines Teils der Belegschaften dabei helfen sollen, den Arbeits-

\footnotetext{
12 Vgl. auch den Betriebsfall eines französischen Medienversands bei Jaehrling et al. 2018.

13 Der Werksleiter aus Fallstudie I (Logistiklager B) bezeichnete den hauseigenen Algorithmus als „Alleinstellungsmerkmal” und stellte ihn als Schlüsselelement für die Optimierung der auf manuellen Schritten beruhenden Wertschöpfung dar.

14 Sobald eine "Containerisierung" in Standardbehältern gelingt, kann die Automatisierung jedoch Sprünge machen wie das bei der Einführung der Fracht- und Schiffscontainer zu beobachten war. In Logistiklagern wird diese Standardisierung über "Taschensortierer" getestet. Hierbei werden Waren in Hängetaschen gelagert, die platzsparend sind und sich den Proportionen des eingelagerten Produkts anpassen.
} 
kraftengpässen und dem damit einhergehenden Druck zur Aufwertung der Arbeit entgegenzuwirken.

\subsection{Arbeitspolitischer Fix:}

von offenen Beschäftigungssystemen

zu einer wachsenden Stammbelegschaft?

Trotz der erwähnten Ansätze, der Arbeitskräfteknappheit durch die räumliche Ausweitung der Rekrutierung und durch technische Lösungen entgegenzuwirken, stoßen die offenen Beschäftigungssysteme bei Unternehmen der Handelslogistik zunehmend an Grenzen.

Knappheit auf dem Arbeitsmarkt bedeutet für die Arbeiter*innen in der Handelslogistik nichts anderes als gestiegene Machtressourcen, um die häufig formulierten arbeitspolitischen Forderungen in Problemfeldern wie Entgelt, Befristung, Leistungsdruck, Arbeitszeitregelung und Gesundheitsschutz durchzusetzen - sofern die organisatorischen und institutionellen Ressourcen vorhanden sind, um die neue Marktmacht zu kanalisieren. Wie dies geschehen kann, zeigt die Fallstudie eines ostdeutschen Versandzentrums von Jaehrling (2019), in dem der Betriebsrat die Betriebsvereinbarung über flexible Arbeitszeitanpassung vor wenigen Jahren neu ausgehandelt hat. Infolgedessen nimmt der Betriebsrat nun auch aktiver sein Recht wahr, über den Einsatz von Leiharbeit mitzubestimmen.

Ein weiteres Beispiel für ein verändertes Kräfteverhältnis zwischen Beschäftigten und Management ist das Logistiklager A des Online-Versandhändlers in den neuen Bundesländern. Wie wir aus Experteninterviews wissen, wurde - zuerst gewerkschaftlich begleitetet und sorgsam vorbereitet - ein Betriebsrat eingerichtet, der im Anschluss eine Entfristungsstrategie entwickelte. Befristete Neueinstellungen wurden blockiert, solange im Gegenzug nicht auch Kolleg*innen entfristet wurden. Ebenso verweigerte der Betriebsrat der Anordnung von Überstunden seine Zustimmung, um im Tausch dafür das Management zu Neueinstellungen zu bewegen und so die persönliche Belastung durch Mehrarbeit zu begrenzen. Im Ergebnis stieg der Anteil der entfristeten Kernbelegschaft im Unternehmen von ca. 40 auf $70 \%$ an. Die Aussagen des Managements von Logistiklager B weisen darauf hin, dass der Druck steigt, auf die neue Arbeitsmarktlage mit einer veränderten betrieblichen Beschäftigungspolitik zu reagieren. Auch aus Managementsicht ist es nun von Bedeutung, Mitarbeiter ${ }^{*}$ innen an den Betrieb zu binden, denn die extreme Fluktuation der Belegschaften in manchen Unternehmen - der Betriebsrat im Logistiklager B (ca. 3000 Beschäftigte) berichtete von der Notwendigkeit, pro Woche 50 bis 100 neue Beschäftigte einzuarbeiten scheint im neuen Arbeitsmarktkontext kaum mehr aufrechterhalten werden zu können.

\section{Arbeitspolitisches Gelegenheitsfenster}

Die managementgetriebenen Strategien - der technologische und sozialräumliche Fix - stehen in Spannung mit der beschäftigtengetriebenen Strategie, die Arbeit aufzuwerten. Welcher Fix zu welchem Grad für die Stabilisierung des Produktionsmodells eingesetzt werden wird, hängt somit vom Kräfteverhältnis der sozialen Akteure im Betrieb ab. Zusätzlich stoßen die Strategien auf interne Widersprüche und Grenzen.

Erstens wird die systematische Anwerbung nicht-deutschen Personals kaum ausreichen, um jenen Arbeitskräfteüberschuss wiederherzustellen, der für die 199oer und 2oooer Jahre charakteristisch war (sozialräumlicher Fix), sondern eher als ein Flexibilitätspuffer durch Leiharbeit fungieren. Auch im Ausland angeworbene Arbeiter*innen formulieren derzeit höhere Ansprüche an ihren Arbeitsplatz und verfügen über diverse Möglichkeiten, zwischen Alternativoptionen auszuwählen. Im Logistiklager A waren es beispielsweise besonders die polnischen Beschäftigten, die sich aktiv für die Gründung eines Betriebsrats engagierten - gerade weil sie in der Vertretung eine Chance sahen, die unsozialen Arbeitszeitregelungen für sie als Pendler*innen zu verbessern.

Zweitens hat die Strategie, Arbeitskraft durch neue Automatisierungslösungen zu substituieren, ihre Grenzen. Derartige Langzeitinvestitionen (sofern sie technologisch überhaupt praxisfähig sind) sind angesichts der intensiven Konkurrenzsituation der Handelslogistik ein riskantes Unterfangen für jeden Betrieb und erfordern darüber hinaus eine gute Kapitalausstattung.

Welche Aussichten hat dann drittens der arbeitspolitische Fix? Zwar kann diese Strategie an dem Eigeninteresse der Betriebe ansetzen, ihre Personalausstattung zu sichern. Doch auch für die arbeitspolitische Lösung der instabilen betrieblichen Situation treten Gegentendenzen auf den Plan. Befristung und Leiharbeit sind kein Auslaufmodell. Sie bleiben, flankiert von teils gewerkschaftsfeindlichen Haltungen sowie wenig Konflikterfahrung in der Branche, ein effektives Mittel der Logistikunternehmen, um ihre Verhandlungsmacht in Lohnkämpfen zu behaupten. Denn erfahrungsgemäß tragen vor allem die entfristeten Beschäftigten die Streiks und Mobilisierungen (Apicella 2016). Der jahrelange Tarifkampf zwischen der Dienstleistungsgewerkschaft ver.di und Amazon zeigt, dass gestiegene Marktmacht allein nicht ausreicht, um beispielsweise einen Tarifvertrag zu erzwingen.

Inwiefern die Logistikarbeiter*innen die soziale Stabilität des Produktionsmodells in der Handelslogistik aufkündigen und in betrieblichen und sektoralen Kämpfen zu ihren Gunsten neu aushandeln, wird auch davon abhängen, ob es den Gewerkschaften in diesem Gelegenheitsfenster gelingt, ihre aktive Mitgliederbasis zu erweitern 
und demokratisch einzubeziehen. Ebenso kann ein politisches Umfeld hilfreich sein, in dem beispielsweise das Disziplinarregime der aktivierenden Arbeitsmarktpolitik abgebaut und Strukturpolitik mit dem Ziel einer Aufwertung der Arbeitsbedingungen verknüpft werden. Unter diesen Umständen könnte die Logistik durchaus eine Branche werden, in der das „Dienstleistungsproletariat“ (Staab 2014) neue Formen der Kollektivität und der arbeitspolitischen Militanz ausbildet. Arbeitspolitische Erfolge in der Handelslogistik könnten so zum Vorbild für das gesamte Feld der einfachen Dienstleistungen werden.

\section{LITERATUR}

Apicella, S. (2016): Amazon in Leipzig. Von den Gründen, (nicht) zu streiken, Berlin

BA (Bundesagentur für Arbeit) (2019a): Arbeitslosenquote aller zivilen Erwerbspersonen: Bundesländer, Monate, Geschlecht, Nürnberg BA (2019b): Beschäftigte nach Berufen (Klassifikation der Berufe 2010) Deutschland, West/Ost und Länder (Quartalszahlen), Nürnberg BA (2019c): Entgeltatlas, Nürnberg

Barthel, G. / Rottenbach, J. (2017): Reelle Subsumtion und Insubordination im Zeitalter der digitalen Maschinerie. Mituntersuchung der Streikenden bei Amazon in Leipzig, in: PROKLA Nr. 187, 47 (2), S. 249-270

Bonacich, E./ Wilson, J. B. (2008): Getting the goods: Ports, labor, and the logistics revolution, Ithaca, NY

Boyer, R./ Freyssenet, M. (2003): Produktionsmodelle. Eine Typologie am Beispiel der Automobilindustrie, Berlin

Briken, K./ Taylor, P. (2018): Fulfilling the "British way": beyond constrained choice - Amazon workers' lived experiences of workfare, in: Industrial Relations Journal 49 (5-6), S. 438-458

Brynjolfsson, E./ McAfee, A. (2014): The second machine age. Work, progress, and prosperity in a time of brilliant technologies, New York

Butollo, F. / Engel, T. / Füchtenkötter M. / Koepp, R. / Ottaiano, M. (2018): Wie stabil ist der digitale Taylorismus? Störungsbehebung, Prozessverbesserungen und Beschäftigungssystem bei einem Unternehmen des Online-Versandhandels, in: Arbeits- und Industriesoziologische Studien 11 (2), S. 143-59

BVL (Bundesvereinigung Logistik) (2017): Fachkräftemangel in der Logistik. Eine Umfrage der Bundesvereinigung Logistik (BVL) e. V., Bremen

Coe, N. (2014): Missing links: Logistics, governance and upgrading in a shifting global economy, in: Review of International Political Economy 21 (1), S. 224-256 Dörre, K./ Haubner, T. (2012): Landnahme als Bewährungsproben - Ein Konzept für die Arbeitssoziologie, in: Dörre, K. / Sauer, D. / Wittke, V. (Hrsg.): Kapitalis mustheorie und Arbeit. Neue Ansätze soziologischer Kritik, Frankfurt a. M./ New York, S. 63-108

Dörre, K. / Scherschel, K. / Booth, M. / Haubner, T. / Marquardsen, K. / Schierhorn, K. (Hrsg.) (2013): Bewährungsproben für die Unterschicht? Soziale Folgen aktivierender Arbeitsmarktpolitik, Frankfurt a. M. / New York

Fernie, J./ Sparks, L. (Hrsg.) (2014): Logistics and retail management. Emerging issues and new challenges in the retail supply chain, London

Goes, T. / Schmalz, S. / Thiel, M. / Dörre, K. (2015): Gewerkschaften im Aufwind? Stärkung gewerkschaftlicher Organisationsmacht in Ostdeutschland, Frankfurt a. M.

Harvey, D. (2006): The limits to capital, London/New York

Hirsch-Kreinsen, H./ Karačić, A./ FGW (Hrsg.) (2018): Logistikarbeit in der digitalen Wertschöpfung. Perspektiven und Herausforderungen für Arbeit durch technologische Erneuerungen, Düsseldorf

Ittermann, P./ Eisenmann, M. (2018): Digitalisierung von Einfacharbeit in der Produktion und Logistik, in: Hirsch-Kreinsen, H. / Karačić, A./ FGW (Hrsg.),

a. a. O., S. 57-76

Jaehrling, K. (Hrsg.) (2018): Virtuous circles between innovations, job quality and employment in Europe? Case study evidence from the manufacturing sector, private and public service sector, o. O.

Jaehrling, K. (2019): Amazon ist kein Vorreiter. Zu den Tiefenstrukturen des „Digitalen Taylorismus“ und verbleibenden Spielräumen kollektiver Interessenaushandlung, in: Industrielle Beziehungen 26 (2), S. 169-188
Jaehrling, K. / Gautié, J. / Keune, M./ Koene, B. / Perez, C. (2018): The digitisation of warehousing work. Innovations, employment and job quality in French, German and Dutch retail logistics companies, in: Jaehrling, K. (Hrsg.), a. a. O. S. 281-332

Krause, A. (2012): Bedingungen und Grenzen marktförmiger Beschäftigungsbeziehungen. Eine Exploration offener betrieblicher Beschäftigungssysteme, in: Krause, A. / Köhler, C. (Hrsg.): Arbeit als Ware. Zur Theorie flexibler Arbeitsmärkte, Bielefeld, S. 117-139

Min, H. (2007): Examining sources of warehouse employee turnover, in: International Journal of Physical Distribution \& Logistics Management 37 (5), S. 375-388

Moody, K. (2017): On new terrain. How capital is reshaping the battleground of class war, Chicago

Moody, K. (2018): High tech, low growth: Robots and the future of work, in Historical Materialism 26 (4), S. 3-34

Mulholland, K./ Stewart, P. (2014): Workers in food distribution: Global commodity chains and lean logistics, in: New Political Economy 19 (4), S. 534-558 Newsome, K./ Thompson, P. / Commander, J. (2013): “You monitor performance at every hour": labour and the management of performance in the supermarket supply chain, in: New Technology, Work and Employment 28 (1), S. 1-15 Ortmann, U./ Walker, E.-M. (2019): Arbeitsfolgen der Digitalisierung in der Handelslogistik jenseits von Substitution und Aufwertung. Forschungsbericht zum Projekt "Digitale Arbeitsbedingungen in der Intralogistik des Handels (DiALog)". Forschungsinstitut für gesellschaftliche Weiterentwicklung (FGW): FGW-Studie: Digitalisierung von Arbeit 17, Düsseldorf

Pfeiffer, S. (2016): Bildung und Intralogistik in der Industrie 4.0 - eine empirische Annäherung, in: Arbeit 25 (3-4), S. 195-215

Schmalz, S. / Hinz, S. / Hasenohr, A. / Singe, I. (2017): Totgesagte leben länger: Demografischer Wandel und Arbeitspolitik in Ostdeutschland, in: Berliner Debatte Initial 28 (3), S. 7-21

Seeck, S. / Groß, W./ Bötel, M. / Herrmansdörfer, M. (2014): Logistik im Handel. Strukturen, Erfolgsfaktoren, Trends, Berlin

Silver, B. J. (2003): Forces of labor: workers' movements and globalization since 1870, Cambridge/New York

Staab, P. (2014): Macht und Herrschaft in der Servicewelt, Hamburg Staab, P./ Nachtwey, O. (2015): Die Avantgarde des digitalen Kapitalismus, in Mittelweg 36. Zeitschrift des Hamburger Instituts für Sozialforschung 24 (6), S. 59-84

Vahrenkamp, R. (2011): Die logistische Revolution. Der Aufstieg der Logistik in der Massenkonsumgesellschaft, Frankfurt a. M.

Wagner, B. / Hassel, A. (2017): Arbeitsmigration oder Auswanderung? Eine Analyse atypischer Arbeitsmigration nach Deutschland, in: WSI-Mitteilungen 70 (6), S.409-420, https://www.boeckler.de/wsimit_2017_06_wagner.pdf

Wright, C. / Lund, J. (2006): Variations on a lean theme: work restructuring in retail distribution, in: New Technology, Work and Employment 21 (1), S. 59-74 Zanker, C. (2018): Branchenanalyse Logistik. Der Logistiksektor zwischen Globalisierung, Industrie 4.0 und Online-Handel, Düsseldorf

\section{AUTOREN}

FLORIAN BUTOLLO, Dr., Leiter der Forschungsgruppe „Arbeiten in hochautomatisierten digital-hybriden Prozessen“ am Weizenbaum Institut für die vernetzte Gesellschaft/Wissenschaftszentrum Berlin für Sozialforschung. Forschungsschwerpunkte: Künstliche Intelligenz und Arbeit, Industrielles Internet, Arbeit in globalen Produktionsnetzwerken.

florian.butollo@wzb.eu

ROBERT KOEPP, Wissenschaftlicher Mitarbeiter in der Forschungsgruppe "Arbeiten in hochautomatisierten digital-hybriden Prozessen" am Weizenbaum Institut für die vernetzte Gesellschaft/Wissenschaftszentrum Berlin für Sozialforschung. Forschungsschwerpunkte: Arbeit und Beschäftigung in der Logistik, digitale Rationalisierung von Arbeitsprozessen.

robert.koepp@wzb.eu 